\title{
Nonlinear thermal convection in a layer of nanofluid under G-jitter and internal heating effects
}

\author{
B. S. Bhadauria ${ }^{1, a}$, Palle Kiran ${ }^{1}$ and M. Belhaq ${ }^{2}$ \\ 1 Department of Applied Mathematics, School for Physical Sciences, Babasaheb Bhimrao Ambedkar University, \\ Lucknow-226025, India. \\ 2 Laboratory of Mechanics, Faculty of Science Ain Chock, University Hassan II, B.P. 5366, Maarif, Casablanca, Morocco.
}

\begin{abstract}
This paper deals with a mathematical model of controlling heat transfer in nanofluids. The time-periodic vertical vibrations of the system are considered to effect an external control of heat transport along with internal heating effects. A weakly non-linear stability analysis is based on the five-mode Lorenz model using which the Nusselt number is obtained as a function of the thermal Rayleigh number, nano-particle concentration based Rayleigh number, Prandtl number, Lewis number, modified diffusivity ratio, amplitude and frequency of modulation. It is shown that modulation can be effectively used to control convection and thereby heat transport. Further, it is found that the effect of internal Rayleigh number is to enhance the heat and nano-particles transport.
\end{abstract}

Keywords: Gravity modulation · Rayleigh-Bénard convection · Nanofluid · Internal heating · Nonlinear theory.

\section{Introduction:}

The cooling and heating effects are required by power manufacturing, transportation, electronics etc. These cooling and heating techniques are greatly needed for high energy device. It was clear that common fluids have limited heat transfer capabilities due to their low heat transfer capacity. However some metals are found to have very high thermal conductivity, may be three to four times higher than the common fluids. Therefore it is required to make a substance by combining these two, which will behave like a fluid and have thermal conductivity of a metal. Nanofluids are such substances, made by suspending the nanoparticles (with diameter 1 to 100 nanometer) in the common fluids, called base fluids. A very small amount of these nanoparticles in the base fluids increases the thermal conductivity of the fluids by $15-40 \%$.

A fluid with suspended nano-sized material or material oxide particles is known as nanofluid, Choi [1]). The presence of suspended particles in the base fluid increases the thermal conductivity of the fluid, Masuda et al. [2] and Das et al. [3]. This increment phenomenon of fluid conductivity has many applications in engineering and science, such as in advanced nuclear systems and nano-drugs, Buongiorno and $\mathrm{Hu}$ [4], and Kleinstreuer et al. [5]. Although these particles improved the thermal conductivity of the fluid, they created other problems such as settling, producing drastic pressure drops, clogging channels, and premature wear on channels and components. However, nano-sized particles have an advantage over milli and micro-sized particles because they approach the size of the molecules in the

\footnotetext{
a e-mail: mathsbsb@yahoo.com
}

fluid. This helps the nanoparticles not settling down due to gravity and thus avoid clogging and wearing of channels. A comprehensive review of heat transport in nanofluids is due to Eastman et al. [6]. In spite of several reported studies, it is a fact that no satisfactory explanation could be found so far for abnormal enhancement in the thermal conductivity and viscosity of the fluid due to the presence of nano-particles.

Natural convection in nanofluids have been investigated by many authors in recent past for different physical configurations; Buongiorno [7], Kuznetsov and Nield [8]-[9], Nield and Kuznetsov [10]-[11], Bhadauria and Agarwal [12]-[13]. It is a common finding of these studies that the presence of nano-particles in the base fluid can advance or delay the onset of convection, and thereby decrease or increase heat transport, depending on the concentration gradient of the nano-particles. One interesting findings of these studies is that the instability is purely due to buoyancy force coupled with conservation of nano-particles and is independent of Brownian motion and thermophoresis effects. In fact, Brownian motion and thermophoresis produce their effect only in coupling the temperature and the particle concentration.

Regulation of convective flows by external means such as gravity or temperature modulation has been of great interest due to it's practical applications in various heat and mass transfer problems. Venezian [14] reported the effect of modulated boundary temperatures which gives rise to an unsteady basic temperature gradient on the onset of Rayleigh-Bénard convection. The effect of gravity modulation on Rayleigh-Bénard convection was first reported by Gresho and Sani [15]. These studies introduced external mechanisms to control the convective flow effectively. Following their work, many investigators studied the linear and nonlinear stability of Rayleigh-Bénard convection for different configurations and by using various methods (see Getling [16], Davis [17] and reference therein). The 


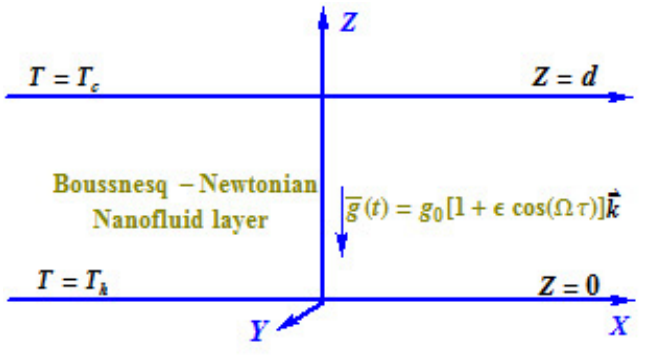

Fig.l : Physical configuration of the problem

effect of temperature modulation on nanofluid convection in porous medium has been reported recently by Umavathi [18] by performing a linear stability analysis.

It appears that all of the above studies deal with the case of uniform heating from below, and the case of internal heating has been largely neglected. There is one paper that deals with an internal heating problem and that is Royer and Flores [19]. This involved a numeral treatment of convection in a two-dimensional box. The case of a homogeneous porous medium with uniform volumetric heating was first studied by Gasser and Kazimi [20] and Nield and Bejan [21]. The literature on internal heating is much less extensive than that on external heating. Some latest studies are due to Bhadauria et al. [22], Bhadauria [23] and Bhadauria et al. ([24],[25],[26]).

There is no study available which concern the effect of g-jitter and internal heating Rayleigh-Bénard convection in nanofluids. This motivates us to do the present study by performing a weakly nonlinear analysis under gravity modulation and internal heating effects.

\section{Governing Equations}

We consider a layer of nanofluid, confined between two horizontal boundaries, respectively at $z=0$ and $z=\mathrm{d}$, heated from below and cooled from above. The fluid layer is extended infinitely in $x$ and $y$-directions, and $z$-axis is taken vertically upward with the origin at the lower boundary. $T_{h}$ and $T_{c}$ are the temperatures at the lower and upper walls respectively such that $T_{h}>T_{c}$. The physical configuration of the nanofluid layer given in Fig.1. Employing the Oberbeck-Boussinesq approximation, the governing equations, to study the thermal instability in a nanofluid layer, are [Buongiorno ([6]):

$$
\begin{array}{r}
\nabla \cdot \mathbf{v}=\mathbf{0}, \\
\rho\left(\frac{\partial \mathbf{v}}{\partial \tau}+\mathbf{v} \cdot \nabla \mathbf{v}\right)+\nabla p=\mu \nabla^{2} \mathbf{v} \\
+\left[\phi \rho_{p}+(1-\phi)\left\{\rho_{f}\left(1-\beta\left(T-T_{c}\right)\right)\right\}\right] \mathbf{g} \\
\frac{\partial T}{\partial \tau}+\mathbf{v} \cdot \nabla T=\kappa_{T} \nabla^{2} T+\frac{(\rho c)_{p}}{(\rho c)_{f}}\left[D_{B} \nabla \phi \cdot \nabla T\right. \\
\left.+\frac{D_{T}}{T_{c}} \nabla T \cdot \nabla T\right]+Q\left(T-T_{c}\right) \\
\frac{\partial \phi}{\partial \tau}+\frac{1}{\epsilon} \mathbf{v} \cdot \nabla \phi=D_{B} \nabla^{2} \phi+\frac{D_{T}}{T_{c}} \nabla^{2} T \\
\mathbf{g}=g_{0}(1+\epsilon \cos (\Omega \tau)) \hat{k}
\end{array}
$$

the variables that are defined in the above equations are given in Nomenclature. Assuming temperature and volumetric fraction of the nanoparticles to be constant at the stress-free boundaries, we may take the boundary conditions on $T$ and $\phi$ as:

$$
\begin{aligned}
& \mathbf{v}=0, \quad T=T_{h}, \phi=\phi_{0} \text { at } z=0, \\
& \mathbf{v}=0, \quad T=T_{c}, \phi=\phi_{1} \text { at } z=d,
\end{aligned}
$$

where $\phi_{1}$ is greater than $\phi_{0}$. The dimensionless variables are considered as given below:

$\left(x^{*}, y^{*}, z^{*}\right)=(x, y, z) / d, \tau^{*}=\tau \kappa_{T} / d^{2},\left(u^{*}, v^{*}, w^{*}\right)=(u, v, w) d / \kappa_{T}$, $p^{*}=p d^{2} / \mu \kappa_{T}, \phi^{*}=\frac{\phi-\phi_{0}}{\phi_{1}-\phi_{0}}$ and $T^{*}=\frac{T-T_{c}}{T_{h}-T_{c}}$, where $\kappa_{T}=$ $\frac{k_{f}}{(\rho c)_{f}}$. The non-dimensionalized governing equations are (after dropping the asterisk for simplicity)

$$
\begin{array}{r}
\nabla \cdot \mathbf{v}=0 \\
\frac{1}{P r}\left(\frac{\partial \mathbf{v}}{\partial \tau}+\mathbf{v} \cdot \nabla \mathbf{v}\right)=-\nabla p-\nabla^{2} \mathbf{v} \\
-\mathbf{g}_{m}(R m-R a T+R n \phi) \hat{e_{z}} \\
\frac{\partial T}{\partial \tau}+\mathbf{v} \cdot \nabla T=\left(\nabla^{2}+R_{i}\right) T+\frac{N_{B}}{L e} \nabla \phi \cdot \nabla T \\
+\frac{N_{A} N_{B}}{L e} \nabla T \cdot \nabla T \\
\frac{\partial \phi}{\partial \tau}+\mathbf{v} \cdot \nabla \phi=\frac{1}{L e} \nabla^{2} \phi+\frac{N_{A}}{L e} \nabla^{2} T \\
\mathbf{v}=0, \quad T=1, \phi=0 \text { at } z=0, \text { and } \mathbf{v}=0, \\
T=0, \phi=1 \text { at } z=1,
\end{array}
$$

where $\mathbf{g}_{m}=(1+\epsilon \cos (\Omega t))$. The non-dimensionlized parameters in the above equations are; $N_{A}$ is the modified diffusivity ratio, which is similar to the Soret parameter that arises in cross diffusion in thermal instability. Prandtl number $\operatorname{Pr}=\frac{\mu}{\rho_{f} k_{T}}$

Lewis number $L e=\frac{k_{T}}{D_{B}}$.

Modified diffusivity ratio

$N_{A}=\frac{D_{T}\left(T_{h}-T_{c}\right)}{D_{B} T_{c}\left(\phi_{1}-\phi_{0}\right)}$

Modified particle-density increment

$N_{B}=\frac{\varepsilon(\rho c)_{p}\left(\phi_{1}-\phi_{0}\right)}{(\rho c)_{f}}$

Thermal Rayleigh-Darcy number

$R a=\frac{\rho g_{0} \beta K d\left(T_{h}-T_{c}\right)}{\mu k_{T}}$

Basic density Rayleigh number

$R m=\frac{\left[\rho_{p} \phi_{0}+\rho\left(1-\phi_{0}\right)\right] g_{0} K d}{\mu k_{T}}$

Concentration Rayleigh number

$R n=\frac{\left(\rho_{p}-\rho\right)\left(\phi_{1}-\phi_{0}\right) g_{0} K d}{\mu k_{T}}$. At the basic state, the nanofluid is assumed to be at rest, therefore the quantities at the basic state will vary only in $z$-direction, and are given by:

$$
\mathbf{v}=0, p=p_{b}(z), T=T_{b}(z), \phi=\phi_{b}(z) .
$$

Substituting Eq.(14) in Eq.(10) and Eq.(11), we get:

$$
\frac{d^{2} T_{b}}{d z^{2}}+R_{i} T_{b}+\frac{N_{B}}{L e} \frac{d \phi_{b}}{d z} \frac{d T_{b}}{d z}+\frac{N_{A} N_{B}}{L e}\left(\frac{d T_{b}}{d z}\right)^{2}=0 .
$$

Using an order of magnitude analysis, Kuznetsov and Nield ([27]), Tzou ([28]-[29]) showed that the second and third terms in equation Eq.(15) are small and hence we have:

$$
\frac{d^{2} T_{b}}{d z^{2}}+R_{i} T_{b}=0, \quad \frac{d^{2} \phi_{b}}{d z^{2}}=0
$$


The boundary conditions for solving Eq.(16) can be obtained from Eq.(13) as:

$$
T_{b}=1, \phi_{b}=0 \text { at } z=0, T_{b}=0, \phi_{b}=1 \text { at } z=1 \text {. }
$$

Solving Eq.(16), subject to the above conditions (17) and (17), we obtain:

$$
T_{b}=\frac{\sin \sqrt{R_{i}}(1-z)}{\sin \sqrt{R_{i}}}, \phi_{b}=z
$$

\section{Stability Analysis}

We now superimpose perturbations on the basic state as given below:

$$
\mathbf{v}=\mathbf{v}^{\prime}, p=p_{b}+p^{\prime}, T=T_{b}+T^{\prime}, \phi=\phi_{b}+\phi^{\prime} .
$$

Substituting the above expression (19) in Eqs.(8)-(11), and using the expressions (18) and (18), eliminating the pressure and introducing the stream function, we obtain:

$$
\begin{array}{r}
\frac{1}{\operatorname{Pr}} \frac{\partial}{\partial \tau}\left(\nabla^{2} \psi\right)-\nabla^{4} \psi=\frac{1}{\operatorname{Pr}} \frac{\partial\left(\psi, \nabla^{2} \psi\right)}{\partial(x, z)} \\
+\left(\operatorname{Rn} \frac{\partial \phi}{\partial x}-\operatorname{Ra} \frac{\partial T}{\partial x}\right) \mathbf{g}_{m} \mathbf{k} \\
-\frac{\partial T_{b}}{\partial z} \frac{\partial \psi}{\partial x}-\left(\nabla^{2}+R_{i}\right) T=-\frac{\partial T}{\partial \tau}+\frac{\partial(\psi, T)}{\partial(x, z)} \\
-\frac{\partial \psi}{\partial x}-\frac{N_{A}}{L e} \nabla^{2} T=\frac{1}{L e} \nabla^{2} \phi-\frac{\partial \phi}{\partial \tau}+\frac{\partial(\psi, \phi)}{\partial(x, z)}
\end{array}
$$

A local nonlinear stability analysis shall be performed and hence we will take the following Fourier expressions:

$$
\begin{gathered}
\psi=\sum_{n=1}^{\infty} \sum_{m=1}^{\infty} A_{m n}(\tau) \sin (m \alpha x) \sin (n \pi z) \\
T=\sum_{n=1}^{\infty} \sum_{m=1}^{\infty} B_{m n}(\tau) \cos (m \alpha x) \sin (n \pi z) \\
\phi=\sum_{n=1}^{\infty} \sum_{m=1}^{\infty} C_{m n}(\tau) \cos (m \alpha x) \sin (n \pi z) .
\end{gathered}
$$

We consider modes $(1,1)$ for stream function, and $(0,2)$ and $(1,1)$ for temperature and nanoparticle concentration:

$$
\begin{gathered}
\psi=A_{11}(\tau) \sin (\alpha x) \sin (\pi z) \\
T=B_{11}(\tau) \cos (\alpha x) \sin (\pi z)+B_{02}(\tau) \sin (2 \pi z) \\
\phi=C_{11}(\tau) \cos (\alpha x) \sin (\pi z)+C_{02}(\tau) \sin (2 \pi z),
\end{gathered}
$$

where the amplitudes $A_{11}(\tau), B_{11}(\tau), B_{02}(\tau), C_{11}(\tau)$ and $C_{02}(\tau)$ are functions of time and are to be determined. Substituting equations (26) - (28) in equations (20) - (22) taking the orthogonality condition with the eigenfunctions associated with the considered minimal mode, we get:

$$
\begin{array}{r}
\frac{d A_{11}(\tau)}{d \tau}=\frac{\operatorname{Pr}}{\delta^{2}}\left\{\alpha ( 1 + \epsilon \operatorname { c o s } ( \Omega t ) ) \left[\operatorname{Rn} C_{11}(\tau)\right.\right. \\
\left.\left.-\operatorname{Ra} B_{11}(\tau)\right]-\delta^{4} A_{11}(\tau)\right\} \\
\frac{d B_{11}(\tau)}{d \tau}=-\left\{\frac{4 \pi^{2} a}{\left(4 \pi^{2}-R_{i}\right)} A_{11}(\tau)+\delta^{2} B_{11}(\tau)\right. \\
\left.-R_{i} B_{11}(\tau)+\pi \alpha A_{11}(\tau) B_{02}(\tau)\right\}
\end{array}
$$

$$
\begin{array}{r}
\frac{d B_{02}(\tau)}{d \tau}=\frac{\pi \alpha}{2} A_{11}(\tau) B_{11}(\tau)-\left(4 \pi^{2}-R_{i}\right) B_{02}(\tau) \\
+\frac{8 \pi^{2} R_{i}}{9 \pi^{4}-10 \pi^{2} R_{i}-R_{i}^{2}} A_{11}(\tau) \\
\frac{d C_{11}(\tau)}{d \tau}=-\left[\alpha A_{11}(\tau)+\frac{1}{L e} \delta^{2} C_{11}(\tau)+\pi \alpha A_{11}(\tau)\right. \\
\left.C_{02}(\tau)+\frac{N_{A}}{L e} \delta^{2} B_{11}(\tau)\right] \\
\frac{d C_{02}(\tau)}{d \tau}=\frac{\pi \alpha}{2} A_{11}(\tau) C_{11}(\tau)-\frac{4 \pi^{2}}{L e}\left[C_{02}(\tau)\right. \\
\left.+N_{A} B_{02}(\tau)\right]
\end{array}
$$

The above system of simultaneous autonomous ordinary differential equations can be subsequently solved numerically using Mathematic NDSolve, subjected to the chosen suitable initial conditions.

\section{Heat and Nanoparticle Concentration Transport}

The thermal Nusselt number, $N u(\tau)$ is defined as

$$
N u(\tau)=1+\left[\frac{\int_{0}^{2 \pi / \alpha_{c}}\left(\frac{\partial T}{\partial z}\right) d x}{\int_{0}^{2 \pi / \alpha_{c}}\left(\frac{\partial T_{b}}{\partial z}\right) d x}\right]_{z=0}
$$

Substituting equations (18) and (27) in Eq.(34), we get

$$
N u(\tau)=1-2 \pi B_{02}(\tau)
$$

The nanoparticle concentration Nusselt number, $N u_{\phi}(\tau)$ is defined similar to the thermal Nusselt number. Following the procedure adopted for arriving at $N u(\tau)$, one can obtain the expression for $N u_{\phi}(\tau)$ in the form:

$$
N u_{\phi}(\tau)=\left(1-2 \pi C_{02}(\tau)\right)+N_{A}\left(1-2 \pi B_{02}(\tau)\right) .
$$

\section{Results and Discussion}

The nanofluids have an enhanced magnitude of thermal conductivity than normal fluids or metals, being attributed to the presence of nanoparticles in them. Here, we have investigated the effect of gravity modulation in a horizontal fluid layer saturated by a nanofluid with internal heating effect. Using Brinkman model and considering top heavy fluid layer, we performed a weakly nonlinear stability analysis to study the heat transport. It is well known that, we make a nonlinear theory to analyze heat transport, which is not possible by linear stability theory. Moreover external regulations of convection is important in the study of thermal instability, therefore, in this paper we have considered gravity modulation for either enhancing or inhibiting the convective heat transport as is required by a real life application. Here note that according to Buongiorno ([7]), for most nanofluids investigated so far $L e$ is large, but as per Bhaduria and Agarwal ([13]) and Umavathi ([18]), we have considered $L e=10$ in the case of nanopartical concentration Rayleigh number. The values of $R_{i}$ are considered to be moderate so that it will not affect the effect of 
gravity modulation of the system by dominating it otherwise. The effect of gravity modulation on heat transport has been depicted in Figs.2-14. The following parameters $R_{i}, \operatorname{Pr}, R n, N_{A}, L e, \epsilon$ and $\Omega$ occurring in the present study, influence the convective heat transport. The first five parameters are related to the nanofluid and the next two parameters concern the external mechanism of controlling convection. Because of small amplitude of modulation, the values of $\epsilon$ are considered to be small. Further, the gravity modulation assumed to be of low frequency, as at low range of frequencies, the effect of frequency on onset of convection as well as on heat transport is maximum. The coefficient of heat transport, i.e. thermal Nusselt number and the coefficient of nanoparticle concentration transport, i.e. concentration Nusselt number are calculated as functions of time and other parameters of the system. The obtained results are depicted in the Figs. 2-14 for $N u(\tau)$ and $N u_{\phi}(\tau)$ verses time $\tau$. In the figures, the values of $N u(\tau)$ and $N u_{\phi}(\tau)$ start with 1 and 2 respectively, and remains constant for a quite some time, showing the conduction state. Then the values of $N u(\tau)$ and $N u_{\phi}(\tau)$ increase as time passes, thus showing that the convection is taking place. These values oscillate and then approach constant values thus showing that the steady state of the system has been achieved.

We fix the values of parameters as $R_{i}=1.0, \operatorname{Pr}=$ 1.0, $R n=10.0, N_{A}=1.0, L e=10.0, \epsilon=0.1$ and $\Omega=2.0$ and drawn the graphs by varying individual values of each parameter. From the Figs. 2 and 3, we observe that the effect of internal heating on thermal instability is destabilizing, as heat transport increases on increasing $R_{i}$. The heat transport is more at higher values of $R_{i}$. This confirms the results obtained most recently by Bhadauria [23] and Bhadauria et al. ([24]-[25]-[26]). ' From the Figs. 4 and 5, we find that initially when time $\tau$ is small the vibrations become of high amplitudes as the value of $\mathrm{Pr}$ increases, and so Nusselt and concentration Nusselt numbers increase, thus increasing the rate of heat and nanoparticle concentration transport. But at large values of time $\tau$, the vibrations become smaller and subsequently the values of $N u$ and $N u_{\phi}$ approach steady state values. The influence of concentration Rayleigh number $R n$ on both thermal Nusselt number as well as on concentration Nusselt number is found to be similar that is to enhance the heat and concentration transport as given in Figs. 6 and 7, which is due to the fact that the nanoparticle concentration is more at the top.

Fig. 8 shows that, $N_{A}$ and $L e$ do not have significant effect on the thermal Nusselt number as reported earlier by Bhadauria and Agarwal ([12]). On the contrary in the case of concentration Nusselt number both $L e$ and $N_{A}$ have increasing effect given in the Figs. 9 and 10 and so the heat and concentration transport.

Figs. 11 and 12 show that the effect of increase in the amplitude of gravity modulation on heat and nanoparticle concentration transport is to increase the values of $\mathrm{Nu}$ and $N u_{\phi}$, and hence transport phenomena in both the cases.

Figs. 13 and 14 show that the effect of increasing frequency of gravity modulation on heat and nanoparticle concentration transport is to decrease the values of $N u$ and $N u_{\phi}$, and hence stabilize the system. Thus, we obtain the classical results as per Gresho and Sani ([15]).

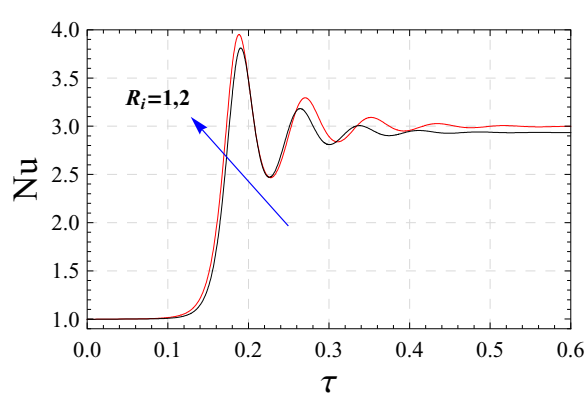

Fig2.Nu vns $\tau$ for different values of $R_{i}$

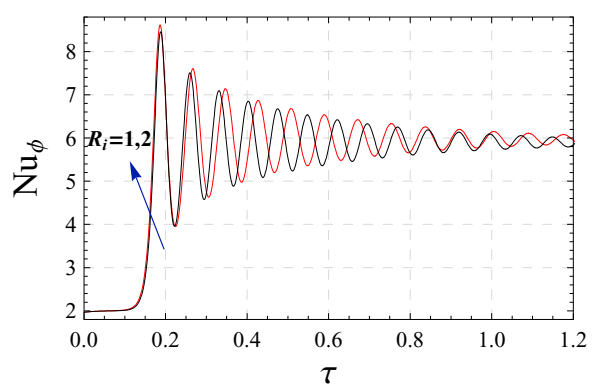

Fig3. $\mathrm{Nu}_{\phi}$ vns $\tau$ for different values of $R_{i}$

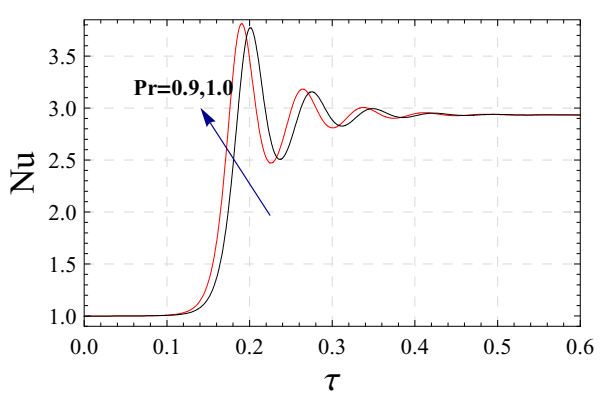

Fig4.Nu vns $\tau$ for different values of $P r$

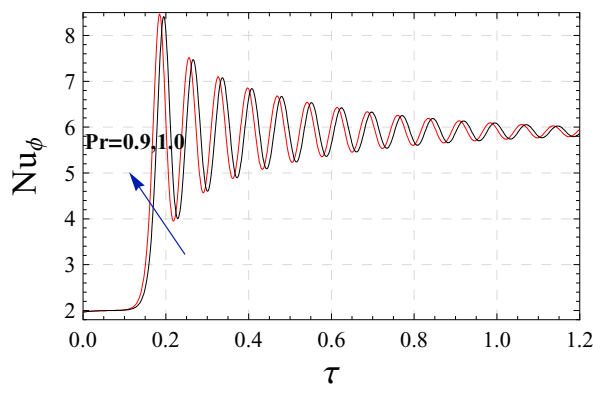

Fig5.Nu ${ }_{\phi}$ vns $\tau$ for different values of Pr

It is observed in most of the cases that there is much effect of parameters on $\mathrm{Nu}$ and $N u_{\phi}$ at low values of time, but less effect at large time, since vibrations become smaller in magnitude, and disappears as $N u, N u_{\phi}$ reach steady state value.

Finally, the parameters $\operatorname{Pr}, \operatorname{Rn}, \epsilon$ have destabilizing effects on the system, while $\Omega$ has stabilizing effect. The parameters $N_{A}$, Le does not show any effect on $N u$, but increase the value of $N u_{\phi}$. 


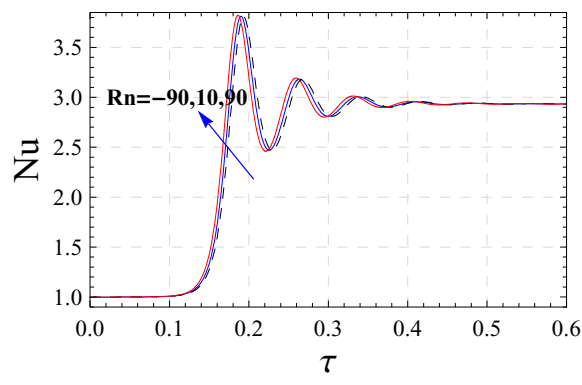

Fig6.Nu vns $\tau$ for different values of Rn

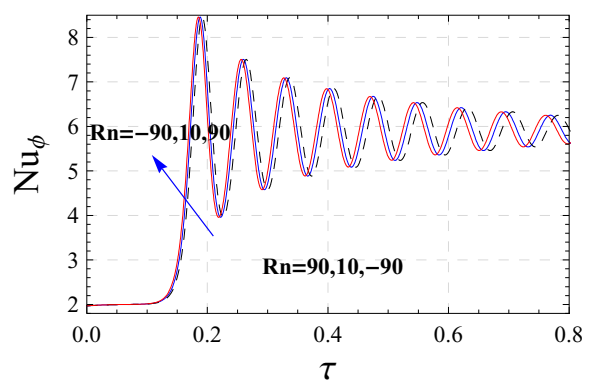

Fig7. $\mathrm{Nu}_{\phi}$ vns $\tau$ for different values of $\mathrm{Rn}$

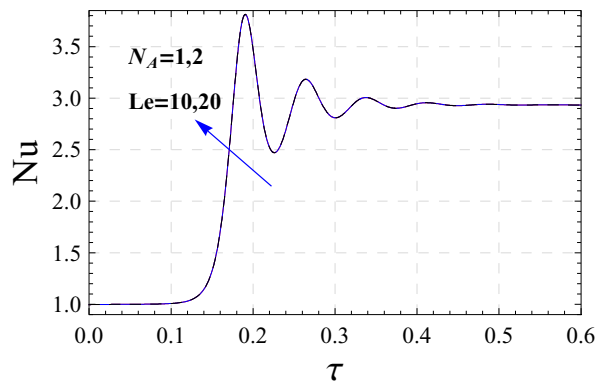

Fig8. Nu vns $\tau$ for different values of Le and $\mathrm{N}_{\mathrm{A}}$

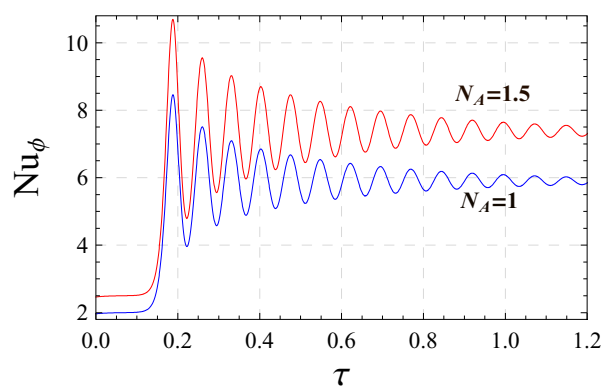

Fig9. $\mathrm{Nu}_{\phi}$ vns $\tau$ for different

\section{Conclusions}

We have investigated weakly nonlinear stability of a vertically vibrating horizontal nanofluid layer with internal heating effect, which is heated from below and cooled from above. Further, we incorporate the effect of Brownian motion along with thermophoresis. The top heavy suspension of nano particles has been considered. The results have been obtained in terms of the concentration and thermal Nusselt numbers with the help of the finite amplitude

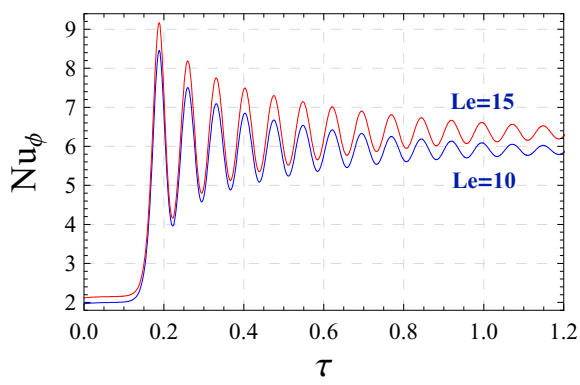

Fig10.Nu ${ }_{\phi}$ vns $\tau$ for different values of Le

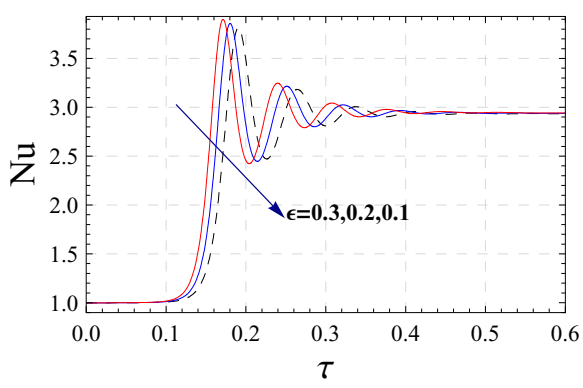

Fig11.Nu vns $\tau$ for different values of $\epsilon$

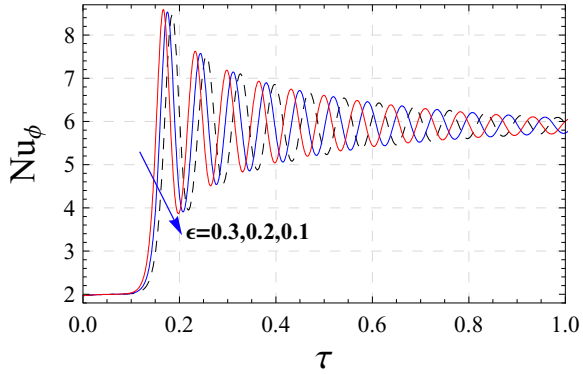

Fig12. $\mathrm{Nu}_{\phi}$ vns $\tau$ for different values of $\epsilon$

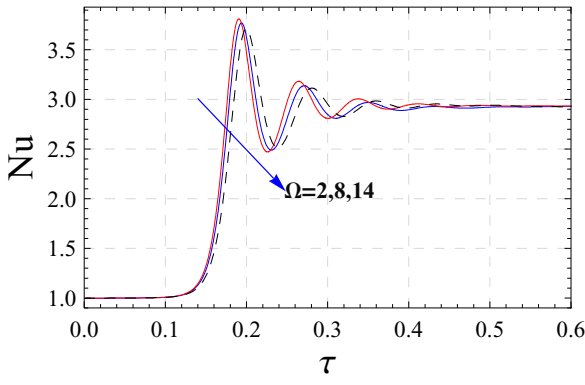

Fig13.Nu vns $\tau$ for different values of $\Omega$

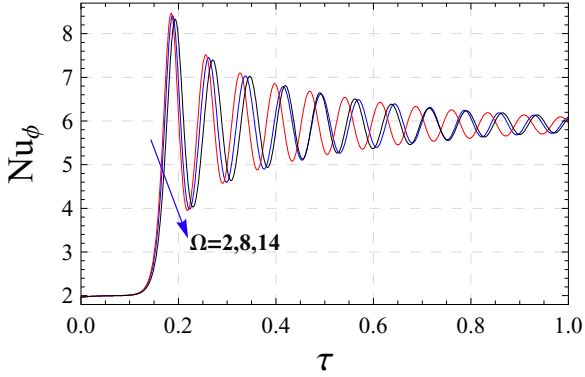

Fg14. $\mathrm{Nu}_{\phi}$ vns $\tau$ for different values of $\Omega$ 
equation. The effect of various parameters have been obtained and depicted graphically. We have the following observations:

1. Gravity modulation can be used to regulate the heat transport effectively.

2. The effect of internal heating is to increase the heat transport in the system.

3 . Increment in concentration Rayleigh number $R n$, modified diffusivity ratio $N_{A}$ and Lewis number $L e$ increase the effect of gravity modulation.

4. An increment in $\mathrm{Pr}$ is to increase the values of $N u(\tau)$ and $N u_{\phi}(\tau)$ at small values of time $\tau$ but no effect at large time $\tau$.

5. The effect of increased nanoparticle concentration $R n$ is to enhance the heat and concentration transport.

6. There is no significant effect of $N_{A}$ and $L e$ on thermal Nusselt number.

7. Increasing $L e, \epsilon, N_{A}$ and $R n$ is to increase the concentration Nusselt number, whereas an increase in $\Omega$ decreases the same, and so the concentration transport.

\section{Nomenclature}

Latin Symbols
$D_{B}$
$D_{T}$
$D a$
$\operatorname{Pr}_{D}$
$Q$
$R_{i}$
$d$
$k_{T}$
$k_{m}$
$p$
$\mathbf{g}$
$\tau$
$T$
$T_{c}$
$T_{h}$
$\mathbf{v}$
$\mathbf{v}_{D}$
$\left(x^{*}, y^{*}, z^{*}\right)$

\section{Greek symbols}

$\epsilon$

$\Omega$

$\alpha$

$\beta$

$\delta$

$\mu$

$\bar{\mu}$

$\rho_{f}$

$\rho_{p}$

$(\rho c)_{f}$

$(\rho c)_{m}$

$(\rho c)_{p}$
Brownian diffusion coefficient

Thermophoretic diffusion coefficient

Darcy number, $D a=\frac{\bar{\mu} K}{\mu d^{2}}$

Prantdl-Darcy number, $\operatorname{Pr}_{D}=\frac{\delta P r}{D a}$

Is the internal heat source

Is the internal Rayleigh number, $R_{i}=\frac{Q d^{2}}{\kappa_{T}}$

Dimensional layer depth

Effective thermal conductivity

Thermal diffusivity of porous medium

Pressure

Modulated gravity field

Time

Temperature

Temperature at the upper wall

Temperature t the lower wall

Nanofluid velocity

Darcy velocity

Cartesian coordinates

Amplitude of modulation

Frequency of modulation

Horizontal wave number

Proportionality factor

Porosity

Viscosity of the fluid

Effective viscosity of the porous medium

Fluid density

Nanoparticle mass density

Heat capacity of the fluid

Effective heat capacity of nanoparticle material Heat. Mass. Transfer., 52 5796-5801, (2009).

\section{References}

1. S. Choi, "Enhancing thermal conductivity of fluids with nanoparticles. in:D.A.Siginer, H.P.Wang(Eds.) Development and applications of non-Newtonian flows,"ASME. FED., 231/MD, 66, 99-105, (1995).

2. H. Masuda, A. Ebata, K. Teramae, N. Hishinuma, "Alteration of thermal conductivity and viscosity of liquid by dispersing ultra fine particles Netsu Bussei,"7, 227-233, (1993).

3. S.K. Das, N. Putra, P. Thiesen, W. Roetzel, "Temperature dependence of thermal conductivity enhancement for Nanofluids,"ASME J. Heat Transfer., 125, 567-574, (2003).

4. J. Buongiorno, W. Hu, "Nanofluid coolant for advanced nuclear power plants,"Paper. No. 5705, Proceedings. ICAPP'05, Seoul., 15-19, (2005).

5. C. Kleinstreuer, J. Li, J. Koo, "Microfluidics of nano-drug delivery,'Int. J. Heat. Mass. Transf., 51, 5590-5597, (2008).

6. J.A Eastman, S.U.S. Choi, W. Yu, L.J. Thompson, "Thermal Transport in Nanofluids,"Annual. Rev. Mater. Res., 34, 219-246, (2004).

7. J. Buongiorno, "Convective transport in nanofluids,"ASME. J. Heat. Transfer., 128, 240-250, (2006).

8. A.V. Kuznetsov, D.A. Nield, "Thermal instability in a porous medium layer saturated by a nanofluid: Brinkman Model,'Transp. Porous. Med., (2009a).

9. A.V. Kuznetsov, D.A. Nield, "Effect of local thermal non-equilibrium on the Onset of convection in porous medium layer saturated by a Nanofluid,"Transp. Porous. Med., 1242-009-9452-8, (2009b).

10. D.A. Nield, A.V. Kuznetsov, "Thermal instability in a porous medium layer saturated by nonofluid,"Int. J. 
11. D.A. Nield, A.V. Kuznetsov, "Onset of convection with internal heating in a weakly heterogeneous porous medium,'”Transp. Porous. Med., 98, 543-552, (2013).

12. B.S. Bhadauria, S. Agarwal, "Natural convection in a nanofluid saturated Rotating porous layer: A Nonlinear Study,'Transp. Porous. Med., 87, 585-602, (2011a).

13. B.S. Bhadauria, S. Agarwal, "Convective transport in a nanofuid saturated porous layer with thermal non equilibrium model,'Transp. Porous Med., 88, 107-131 (2011b).

14. G. Venezian, "Effect of modulation on the onset of thermal convection,”J. Fluid. Mech., 35, 243-254, (1969).

15. P.M. Gresho, R.L. Sani, "The effects of gravity modulation on the stability of a heated fluid layer,'J. Fluid. Mech., 40(4), 783-806 (1970).

16. A.V. Getling, "Rayleigh-Bénard Convection: Structures and Dynamics World Scientific,"Singapore, (1998).

17. S.H. Davis, "The stability of time periodic flows,"Annu. Rev. Fluid. Mech., 8, 57-74, (1976).

18. J.C. Umavathi, "Effect of thermal modulation on the onset of convection in a porous medium layer Saturated by a Nanofluid,"Transp. Porous. Medi., 98, 59-79, (2013).

19. J. Royer, L. Flores, "Two-dimensional naturalconvection in an anisotropic and heterogeneous porous-medium with internal heat-generation"Int. J. Heat. Mass. Transf., 37, 1387-1399, (1994).

20. R. Gasser, M. Kazimi, "Onset of convection in a porous-medium with internal heat generation,"J. Heat Transf. Trans. ASME., 98, 49-54, (1976).

21. D.A. Nield, A. Bejan, "Convection in Porous Media,"4th edn. Springer, New York (2013)

22. B.S. Bhadauria, A. Kumar, J. Kumar, N.C. Sacheti, P. Chandran, "Natural convection in a rotating anisotropic porous layer with internal heat generation,'Transp. Porous. Med., 90, 687-705, (2011).

23. B.S. Bhadauria, "Double diffusive convection in a saturated anisotropic porous layer with internal heat source,'Transp. Porous. Med., 92, 299-320, (2012).

24. B.S. Bhadauria, I. Hashim, P.G. Siddheshwar, "Study of heat transport in a porous medium under G-jitter and internal heating effects,'Transp. Porous. Med., 96, 21-37, (2013).

25. B.S. Bhadauria, I. Hashim, P.G. Siddheshwar, "Effect of internal-heating on weakly non-linear stability analysis of Rayleigh-Bénard convection under gjitter,'Int. J. non-linear. Mech., 54, 35-42, (2013).

26. B.S. Bhadauria, I. Hashim, P.G. Siddheshwar, "Effects of time-periodic thermal boundary conditions and internal heating on heat transport in a porous medium,'Transp. Porous. Med., 97, 185-200, (2013).

27. A.V. Kuznetsov, D.A. Nield, "Thermal instability in a porous medium layer saturated by a nanofluid: Brinkman Model,'Trans. Porous. Med., (2009).

28. D.Y. Tzou, "Instability of nanofluids in natural convection"ASME. J. Heat. Transfer., 130, 072401, (2008a).

29. D.Y. Tzou, "Thermal instability of nanofluids in natural convection'Int. J. Heat. Mass. transfer., 51, 